\title{
Modificación del perfil lipídico sanguíneo en respuesta al ejercicio físico en ratas alimentadas con dietas conteniendo ácidos grasos trans (AGT)
}

\author{
Modification of blood lipid profile in response to exercise in rats fed diets containing trans- \\ fatty acid (TFA) \\ Gloria Tula Bravo-Araujo', Carlos Vilchez Perales
}

\section{RESUMEN}

Objetivo: Evaluar el efecto del ejercicio físico (natación) sobre el perfil lipídico sanguíneo en ratas alimentadas con dietas conteniendo ácidos grasos trans (AGT). Métodos: El tipo de investigación fue experimental. Los datos obtenidos fueron procesados bajo un Diseño Completamente Randomizado con arreglo factorial $2 \times 2$ empleando el procedimiento de análisis de varianza .Se utilizaron 24 ratas Holtzman machos de cuatro meses de edad, con peso promedio de $319 \mathrm{~g}$ y el experimento tuvo una duración total de ocho semanas. Durante la semana uno, 12 ratas recibieron una dieta estándar y las otras 12 ratas recibieron la dieta estándar mezclada con margarina comercial que contenía $5,3 \%$ de AGT. A partir de la semana dos hasta la semana ocho, la mitad (6) de animales de cada grupo fue sometido a uno de los siguientes tratamientos: T1, Dieta estándar-Sedentario; T2, Dieta estándarNatación; T3, Dieta estándar con AGT-Sedentario; T4, Dieta estándar con AGT-Natación. La dieta estándar con AGT contenía 2,9\% AGT. Las ratas de los tratamientos T2 y T4 fueron forzados a nadar por 40 minutos por día durante cinco días por semana. Al término del experimento, se extrajeron muestras de sangre de cada uno de los animales experimentales para determinar las concentraciones séricas de colesterol total (CT), triglicéridos (TGs), HDL-colesterol y LDL-colesterol. Resultados: Con excepción de la concentración de TGs, los otros parámetros no fueron influenciados significativamente $(P>0,05)$ por los tratamientos. La concentración de TGs fue menor $(P<0,05)$ en los grupos de animales que fueron sometidos a actividad física (natación). Conclusiones: El ejercicio físico (natación) disminuye la concentración de TGs sanguíneo en ratas que consumen dietas que contienen ácidos grasos trans.

Palabras clave: AGT, ejercicio físico, perfil lipídico.

\section{ABSTRACT}

Objective: To assess the effect of physical exercise (swimming) on blood lipid profile in rats fed diets containing trans-fatty acids (AGT). Methods: The type of research was experimental. The data obtained were processed under a completely randomized design with a $2 \times 2$ factorial arrangement using the variance analysis procedure. 24 Holtzman rats males from four months of age, with average weight of $319 \mathrm{~g}$ were used and the experiment was a total duration of eight weeks. During one week, 12 rats received a standard diet and the other 12rats received the standard diet mixed with commercial margarine containing 5.3\% of AGT. From week two to week eight, half (6) of animals in each group were subjected to one of the following treatments: T1, Standard-sedentary diet; T2, Standard-swimming diet; T3, standard diet with AGT-sedentary; T4, standard diet with AGT-swimming. The standard diet with AGT contained $2.9 \%$ AGT. The rats of the treatments T2 and T4 were forced to swim for 40 minutes a day for five days per week. At the end of the experiment, blood samples from each of the experimental animals were extracted to determine serum concentrations of total cholesterol (CT), triglycerides (TGs), HDLcholesterol and LDL-cholesterol. Results: With the exception of the concentration of TGs, the other parameters were not influenced ( $\mathrm{P} \& \mathrm{gt} ; 0,05)$ by treatments. The concentration of TGs was lower (P \& It; 0,05 ) in groups of animals that we reubjected to physical activity (swimming). Conclusions: Physical exercise (swimming) decreases the concentration of blood TGs in rats consuming diets containing trans fatty acids.

Keywords: AGT, exercise, lipid profil.

${ }^{1}$ Universidad Nacional Agraria La Molina. Lima, Perú. 


\section{INTRODUCCIÓN}

Estudios de Salud muestran evidencias de que los niveles elevados de colesterol sérico están asociados con enfermedades cardiovasculares y que el origen del problema era el consumo elevado de colesterol y de grasa saturada. Ante esta situación, la industria de alimentos respondió con un aumento en la producción de aceites vegetales parcial o totalmente hidrogenados (Ballesteros-Vásquez, Valenzuela-Calvillo, Artalejo-Ochoa, RoblesSardin, 2012) dando origen a los ácidos grasos trans (AGT). Estos ácidos grasos trans se forman mediante un proceso de hidrogenación que consiste en la introducción de un gas hidrógeno a los aceites vegetales bajo ciertas condiciones de presión y temperatura usando un metal catalizador (Níquel) (Monrroy, 2009), dicho proceso permite otorgarle al producto características deseables como alargar el tiempo de vida útil, evitar la rancidez, mejorar textura, potenciar sabor y por último abaratar costos de producción. Si bien es cierto presenta múltiples ventajas, también tiene inconvenientes, muchos estudios señalan que consumir en exceso alimentos que contengan (AGT), modifican negativamente el perfil lipídico dado que no solo aumentan los niveles de triglicéridos (TG), Colesterol total (CT), y CLDL sino que reducen el C-HDL, por lo que estaría relacionado con el aumento del riesgo de enfermedades cardiovasculares, hipercolesterolemia, hipertrigliceridemia y arteriosclerosis (Ballesteros-Vásquez, Valenzuela-Calvillo, Artalejo-Ochoa, RoblesSardin, 2012). Estas enfermedades son multifactoriales y resultan de la combinación de causas externas e internas.

Se acepta que los malos hábitos en el estilo de vida son las principales causas en el aumento de este padecimiento, donde la inadecuada alimentación junto al sedentarismo, son los que más repercuten en la salud. La prevención se sustentaría en llevar un determinado régimen de vida que incluya ejercicio físico diario y una dieta equilibrada. Estudios científicos señalan los múltiples beneficios del ejercicio aeróbico en la mejora del perfil lipídico por la reducción de la hipercolesterolemia total, triglicéridos (TG), C-LDL y aumento del C-HDL (Alfonso y Ariza, 2008), por lo que se pensó que un programa de ejercicio mejoraría el perfil lipídico que se vería afectado por el consumo de AGT.

Por lo expuesto anteriormente, el objetivo del presente trabajo fue evaluar la modificación del perfil lipídico sanguíneo en respuesta al ejercicio físico en ratas alimentadas con dietas conteniendo AGT, medido por la concentración de TG, CT, C-HDL y C-LDL.

\section{MATERIAL Y MÉTODOS}

La investigación realizada fue de tipo prospectivo-longitudinal, se llevó a cabo en el Bioterio de la Facultad de Zootecnia de la Universidad Nacional Agraria la Molina y tuvo una duración de ocho semanas. Se trabajó con 24 ratas machos de la especie Holtzman de cuatro meses de edad, con peso promedio de $319 \pm 12,34 \mathrm{~g}$, las cuales recibieron alimento y agua ad libitum durante las ocho semanas que duro el estudio y se les dio exclusivamente el alimento mezclado con margarina que contenía $5,29 \%$ de grasa trans.

El experimento fue dividido en dos etapas: En la primera, denominada etapa de acostumbramiento, las 24 ratas machos se dividieron en dos grupos (dieta estándar y dieta estándar con $2,9 \%$ de AGT) y se colocaron en jaulas metálicas individuales, en las jaulas pares se ubicaron las ratas que consumieron la dieta estándar o dieta que habitualmente consumen las ratas en el Bioterio y en las jaulas impares, las ratas que consumieron la dieta con 2,9\% de AGT, por espacio de una semana. La dieta con $2,9 \%$ de AGT se obtuvo mezclando $1800 \mathrm{~g}$ de la dieta estándar con $200 \mathrm{~g}$ de margarina Primavera en una mezcladora HOBART a velocidad mínima del equipo hasta lograr una mezcla homogénea (ausencia de trozos de margarina). La dieta se preparó cada cinco días y se almacenó en un recipiente oscuro y puesto a refrigeración. A ambas dietas se realizó el análisis de perfil de ácidos grasos.

Finalizada la semana de acostumbramiento los animales fueron pesados y se separaron 6 ratas por cada tipo de dieta según su ganancia de peso para que naden. En la segunda, etapa de entrenamiento de los animales, las ratas seleccionadas para la práctica de ejercicio, fueron puestas a nadar en un cilindro de plástico de 1,10 m de largo x $1 \mathrm{~m}$ de ancho $\mathrm{x}$ $46,5 \mathrm{~cm}$ de altura con un volumen de agua de 320 litros aproximadamente. El grupo de entrenamiento se ejercitó de lunes a viernes por espacio de 40 minutos diarios durante siete semanas. Se registró todos los fines de semana el peso de los animales, así mismo; se 
cuantificó la cantidad de dieta diaria consumida por cada uno de los animales.

Al término del periodo experimental los animales fueron anestesiadas con Ket-A-100 y sedados con Dormi-Xyl2 para ser sacrificados y obtener las muestras de sangre, dichas muestras fueron colocadas en tubos vacuette de $4 \mathrm{ml}$ y llevadas al laboratorio de Bioanálisis de la Facultad de Ciencias de la UNALM, donde se centrifugó para la obtención del suero, el mismo que sirvió para medir el C-T, C-HDL, CLDL y TG, por método enzimático; utilizando un Espectofotómetro Biomate marca Thermo y el reactivo de la marca Valtek.

Los datos obtenidos fueron procesados bajo un diseño completamente Randomizado con arreglo factorial $2 \times 2$ empleando el análisis de varianza (ANOVA), para lo cual se usó el programa MINITAB 15.

\section{Modelo estadistico}

Modelo Aditivo Lineal:

$$
Y_{i j k}=\mu+D i+E_{j}+(D E)_{i j}+\varepsilon_{i j k}
$$

Donde:

$\mathrm{Y}_{\mathrm{ijk}} \quad$ : Valor estadístico de cada observación en cada unidad experimental.

$\mu \quad$ : Media general.

$\mathrm{D}_{\mathrm{i}} \quad$ : Efecto del factor $\mathrm{D}$ (dieta).

$\mathrm{E}_{\mathrm{j}} \quad$ : Efecto del factor $\mathrm{E}$ (ejercicio).

$(D E)_{i j}$ :Efecto de la interacción de los tratamientos (en el i-ésimo nivel de D y elj-iésimo nivel E).

$\varepsilon_{\mathrm{ijk}} \quad$ : Efecto aleatorio o error en la obtención de $Y_{\mathrm{ijk}}$.

\section{RESULTADOS}

En la Tabla 1 se observan los resultados del perfil de ácidos grasos para dieta estándar y dieta con ácidos grasos trans, donde la incorporación de margarina "Primavera" en un $10 \%$ aumenta a un 2,9\% los ácidos grasos trans frente a la dieta estándar, los ácidos grasos Saturados y Monoinsaturados aumentaron, y los ácidos grasos Poliinsaturados, W3 y W6 disminuyeron respecto a la dieta estándar.
Tabla 1. Perfil de ácidos grasos de la dieta estándar y la dieta con 2,9\% de Grasa Trans

\begin{tabular}{|c|c|c|}
\hline & \multicolumn{2}{|c|}{ g/100 g grasa } \\
\hline & $\begin{array}{l}\text { Dieta } \\
\text { estándar }\end{array}$ & $\begin{array}{l}\text { Dieta con } 2,9 \% \\
\text { de Grasa Trans }\end{array}$ \\
\hline Ácidos Grasos Saturados. & 17,85 & 38,27 \\
\hline Ácidos Grasos Monoinsaturados & 26,04 & 35,48 \\
\hline Ácidos Grasos Poliinsaturados & 56,11 & 26,25 \\
\hline Ácidos Grasos W3 (Linolénico) & 3,02 & 1,36 \\
\hline Ácidos Grasos W6 (Linoleico) & 53,09 & 24,90 \\
\hline Ácidos Grasos Trans & 0,00 & 2,89 \\
\hline
\end{tabular}

El análisis estadístico de los datos obtenidos en la presente investigación no encontró interacción (Dieta * A.F.) significativa $(p>0,05)$ entre el tipo de dieta y la actividad física de los animales como se aprecia en la Tabla 2, los niveles de TG disminuyeron tanto en la dieta estándar como en dieta estándar con $2,9 \%$ de grasas trans, esta disminución de los niveles de TG fue producto de la práctica de ejercicio (natación) mas no a la dieta.

Tabla 2. Promedio $(\mathrm{mg} / \mathrm{dl})$ de valores lipídico sanguíneo deratas alimentadas con dietas que contienen ácidos grasostrans y sometidas a actividad física (natación).

\begin{tabular}{|c|c|c|c|c|c|c|}
\hline \multirow{2}{*}{ TRAT } & \multirow{2}{*}{ DIETA ${ }^{1}$} & \multirow{2}{*}{ A. $F^{2}$} & \multicolumn{4}{|c|}{ Perfil Lipídico (mg/dl) } \\
\hline & & & T.G. & C-T. & C-HDL & C-LDL \\
\hline 1 & A & $S$ & 127,75 & 70,50 & 50,33 & 6,833 \\
\hline 2 & A & $\mathrm{N}$ & 92,80 & 74,50 & 43,00 & 12,833 \\
\hline 3 & B & S & 118,58 & 77,00 & 48,33 & 7,417 \\
\hline 4 & B & $\mathrm{N}$ & 103,60 & 73,00 & 48,00 & 4,750 \\
\hline \multirow{2}{*}{\multicolumn{2}{|c|}{ Efecto de dieta }} & A & $111^{a}$ & $73^{a}$ & $47^{a}$ & $10^{\mathrm{a}}$ \\
\hline & & B & $111^{a}$ & $75^{\mathrm{a}}$ & $48^{a}$ & $6^{a}$ \\
\hline \multirow{2}{*}{\multicolumn{2}{|c|}{ Efecto de A.F. }} & S & $124^{a}$ & $74^{\mathrm{a}}$ & $49^{a}$ & $7^{\mathrm{a}}$ \\
\hline & & $\mathrm{N}$ & $\begin{array}{c}99^{\mathrm{b}} \\
\text { Probabilida }\end{array}$ & $74^{\mathrm{a}}$ & $46^{a}$ & $g^{a}$ \\
\hline \multicolumn{2}{|l|}{ Dieta } & & 0,925 & 0,538 & 0,634 & 0,165 \\
\hline \multicolumn{2}{|l|}{ A. F. } & & 0,009 & 1,000 & 0,231 & 0,529 \\
\hline \multicolumn{2}{|c|}{ Dieta *A.F. } & & 0,259 & 0,328 & 0,273 & 0,111 \\
\hline
\end{tabular}

Letras minúsculas distintas indican diferencia significativa en las columnas $(p<0,05)$.

La práctica de ejercicio (natación), fue capaz de disminuir los niveles de triglicéridos en un $27,36 \%$ en dieta estándar y un $12,63 \%$ en dieta con $2,9 \%$ de grasa trans, resultando significativo $(p<0,05)$; mientras que para variables $C-T, C-L D L$ y $C-H D L$ no resulto significativo $(p>0,05)$. 


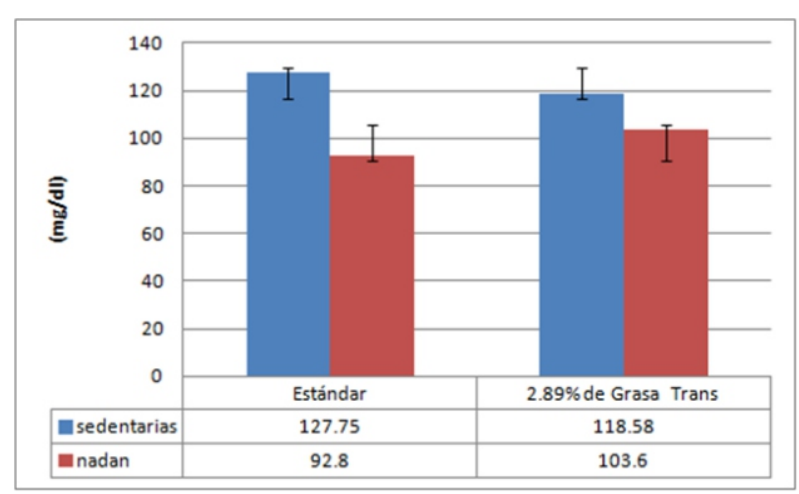

Figura 1. Niveles de Triglicéridos de ratas que consumieron dieta estándar y dieta con $2,89 \%$ de Grasa Trans. Las barras indican la media \pm desviación estándar de los Triglicéridos ( $\mathrm{mg} / \mathrm{dl})$

después de 8 semanas que duró el estudio

Para la dieta estándar $(127,75 \pm 22,53$ y $92,8 \pm 10,84)$; para la dieta estándar con $2.89 \%$ de Grasa Trans $(118,58 \pm 27,00$ y $103,6 \pm 20,43)$ en ratas sedentarias y ratas que practicaron natación respectivamente, donde se observó que la natación logró disminuir los niveles de TG en ambas dietas.

No encontramos diferencia estadísticamente significativa $(p>0,05)$ entre las concentraciones de C-T, C-LDL y C-HDL entre los grupos (Tabla 2 y Figura 2).

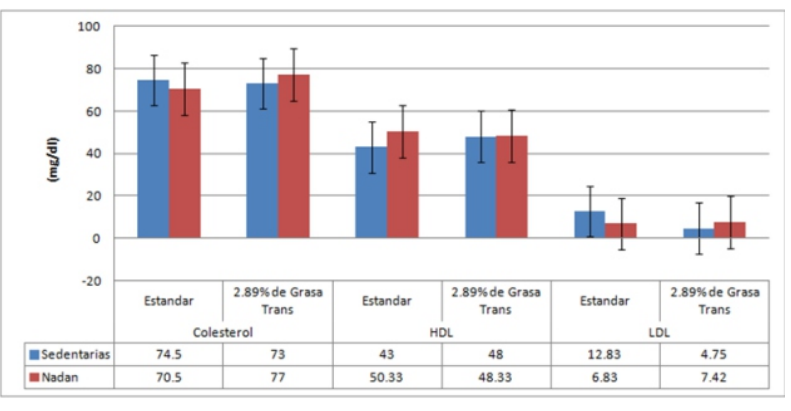

Figura 2. Niveles de Colesterol total, C-HDL, C-LDL de ratas que consumierondieta estándar y dieta con $2,89 \%$ de Grasa Trans. Las barras indican la media \pm desviación estándar de Colesterol total, C-HDL, C-LDL (mg/dl) después de 8 semanasque duró el estudio.

Para la dieta estándar, C-T $(70,5 \pm 11,095$ y $74,5 \pm 11,54)$; C-HDL $(50,33 \pm 10,91$ y $43 \pm 7,9)$; CLDL $(6,83 \pm 2,99$ y $12,83 \pm 10,07)$ para la dieta estándar con $2,89 \%$ de Grasa Trans C$\mathrm{T}(77 \pm 8,72$ y $73 \pm 5,35)$; C-HDL $(48,33 \pm 5,41$ y $48 \pm 3,51)$; C-LDL $(7,42 \pm 3,96$ y $4,75 \pm 5,26)$ en ratas sedentarias y ratas que practicaron natación respectivamente.

\section{DISCUSIÓN}

Los resultados (Tabla 2 y Figura 1 ) indican que la práctica de ejercicio (natación), disminuyó los niveles de triglicéridos en ambas dietas. Estos resultados son coincidentes con los publicados por (LeBlanc y Janssen, 2010) donde muestran una disminución de los niveles de TG y C-HDL mientras que no hubo relación dosis respuesta para C-LDL con ejercicio de moderada a vigorosa actividad. (Aspiroz y Nuviala, 2002) donde se demuestra el descenso de la concentración de TG causada por el ejercicio físico tanto en varones como en mujeres que practicaban diversos deportes respecto al grupo control, formado por individuos sedentarios.

La mayoría de los investigadores concuerdan en que el descenso de TG en plasma después de hacer ejercicio es más significativo cuando la sesión de ejercicio es prolongado (exceso de 30 a 40 minutos). Se confirmó que hay una reducción retrasada de TG plasmáticos que no se produce sino hasta que ocurre un periodo de 30 minutos después de haber hecho ejercicio. Este aumento del catabolismo de TG parece relacionada con el aumento de la lipoproteínLipasa (LPL), que permanece elevada durante varios días tras la sesión de ejercicio (Anderson y Cockayne, 1995 citado por Echevarría, 2006).

La explicación del descenso de los niveles de TG plasmáticos radica en el efecto que tiene el ejercicio físico sobre la actividad de la LPL del músculo esquelético y tejido graso, conjuntamente con la disminución de la acción de la lipasa hepática (Aspiroz y Nuviala, 2002). Coincide con dicha explicación (Alfonso y Ariza, 2008) quienes atribuyen el beneficio que tiene el ejercicio físico sobre el perfil lipídico al aumento de la actividad de la LPL extrahepática, la que origina la reducción de los TG al permitir un incremento de la hidrólisis de TG de la lipoproteína de muy baja densidad (VLDL) y quilomicrones (QM), favoreciendo también un aumento de la HDL al disminuir su intercambio lipídico con esta lipoproteína rica en TG. También (Wong-On y Murillo-Cuzza, 2004) señalan como uno de los efectos principales del ejercicio la disminución de los TG plasmáticos, otorgando esta disminución al aumento de la actividad de la LPL quien rompe la unión VLDL-TG y aumenta el consumo de TG y su uso por el músculo esquelético. Plantea además, que el ejercicio aeróbico está asociado no solo al aumento de la LPL sino también a la LCAT y TG lipasa y a la disminución de la lipasa hepática. 
En cuanto a la concentración de CT, C-HDL y CLDL no se encuentran diferencias significativas entre los tratamientos (Tabla 2 y Figura 2), estos resultados son contrarios a los estudios que mencionan que la práctica de ejercicio realizado en forma regular aumentan los niveles de C-HDL y disminuyen los niveles de CT y C-LDL. Entre las causas que podrían haber ocasionado estos resultados estaría el tipo de alimentación, el estrés, la duración de la práctica de ejercicio (natación), la falta de control sobre la ingesta dietética y a que no se tomó en cuenta la restricción calórica, por lo que se sugiere desarrollar ensayos que combinen ejercicio con los diferentes tipos de dieta, prolongar el tiempo de práctica de ejercicio y controlar la ingesta dietética y calorías.

Los hallazgos descritos sobre los parámetros de CT, C-HDL y C-LDL como consecuencia del efecto del ejercicio son muy dispares. La existencia de resultados discordantes son atribuidos a diversos factores, en el estudio de García-Cardona, Nieto y Landázuri (2015) se otorgó un incremento significativo de CT, CLDL, VLDL y TG y una disminución significativa de C-HDL al estrés, mala nutrición y hábitos no saludables de los estudiantes de Medicina adjudicándole mayor peso sobre la modificación del perfil lipídico que un programa de ejercicio. Dentro del factor de la alimentación el que más influye en el perfil lipídico es la composición de ácidos grasos de la dieta (Alfonso y Ariza, 2008). La grasa está constituida por unos componentes básicos denominados ácidos grasos. Encontramos 3 tipos de ácidos grasos: saturados, monoinsaturados y poliinsaturados; si en la grasa hay un predominio de ácidos grasos saturados, se origina una elevación de los niveles de colesterol en sangre especialmente de la fracción LDL; por el contrario, tanto los ácidos grasos poliinsaturados como monoinsaturados pueden reducir el C-T y CLDL, cuando se reemplazan en la dieta a las grasas saturadas y a los ácidos grasos trans (Alfonso y Ariza, 2008).

Estudios corroboran la importancia de una dieta balanceada para mantener el perfil lipídico adecuado; así por ejemplo, Souki et al. (2007) en Adición de mayonesa a la dieta de rata Sprangue-Dawley incrementa la glicemia y los TG con disminución del C-HDL, afirma que un mayor incremento de TG se observa en dietas con alto contenido de monoinsaturados y que niveles elevados de ácidos grasos poliinsaturados disminuyen la concentración del C-HDL; otro estudio, Poveda et al. (2005) atribuye la disminución del C-HDL en aceite de palma al alto contenido de ácidos grasos saturados. En la dieta estándar, el ácido graso poliinsaturado se encuentra en mayor cantidad $(56,11 \%)$ y en la dieta con $2,9 \%$ de grasa trans, el ácido graso saturado $(38,27 \%)$, de ahí podría explicarse los resultados de disminución de CHDL frente al ejercicio.

Entre otro de los factores que pueden provocar alteraciones en el perfil lipídico está el estrés, Camiletti-Moirón et al. (2015) en su trabajo de investigación "Efectos de un protocolo de entrenamiento de alta intensidad sobre marcadores fisiológicos de estrés en ratas", obtuvo resultados similares a los obtenidos en nuestro estudio, trabajó con 40 ratas divididas en dos grupos experimentales, ratas sedentarias $(n=20)$ y ratas con entrenamiento de alta ntensidad (EAI) $(n=20)$, el grupo experimental fue entrenado siguiendo el protocolo de entrenamiento de alta intensidad basado en el desarrollo de la fuerza de hipertrofia en un tapiz rodante con cargas regulables, el estudio tuvo una duración de 12 semanas con 3-4 sesiones/semana y detectaron valores adversos en algunos marcadores de perfil lipídico en el grupo de EAI como mayor C-LDL plasmático, tan solo los TG, presumiblemente por ser la principal fuente de energía en el ejercicio fueron menores en el grupo de ratas de EAI con respecto a las sedentarias. Este fenómeno lo atribuyó a una posible alteración metabólica provocada por el estrés. Si bien en nuestro caso, no determinamos el estrés como si lo hicieron en dicho estudio, las ratas que nadaron mostraron una disminución en el rendimiento del nado, fatiga después de realizado el ejercicio, irritabilidad y desesperación por salir del agua como manifestaciones de que el ejercicio fue excesivo, lo que podría haber influido en nuestros resultados, obteniendo disminución significativa para TG $(123,125 \pm 24,17$ y $98,13 \pm 16,58)$ en ratas sedentarias y ratas que practicaban natación respectivamente, CT $(73,75 \pm 10,44$ y $73,75 \pm 8,76)$, C-HDL $(48,5 \pm 9,73$ y $46,33 \pm 4,89)$, C-LDL $(7,13 \pm 3,57$ y $8,79 \pm 8,89)$ en ratas sedentarias y ratas que practicaban natación respectivamente, no fueron significativos, pero si se observó una disminución del C-HDL y un aumento del C-LDL frente al ejercicio. 
Salgado, Rivera, Habacuc, et al. (2003) corrobora nuestro resultado referente a los niveles de C-LDL en su estudio realizado "Influencia de la grasa corporal y el sexo sobre la respuesta de los lípidos séricos y el ejercicio físico en personas con diferente capacidad aeróbica" donde no fueron significativos, atribuyendo estos resultados a que se requiere la práctica de un ejercicio prolongado (más de 2 horas) para obtener resultados significativos; para nuestro estudio incrementar más tiempo de nado a las ratas representaría mucho esfuerzo de parte de ellas ya que en pruebas piloto se observó que cuando nadaban más de 40 minutos les sangraba la nariz, pero podría considerarse ampliar el tiempo de práctica de ejercicio físico (natación) a más de siete semanas para ver si se consigue disminuir el CLDL.

Bermejo y Moraleda (2013), reportó disminución significativa en todos los grupos respecto a TG, C-T y C-LDL cuando se les asignó una dieta equilibrada con un $25 \%$ de restricción desarrollando ejercicio físico de fuerza, aeróbico y combinado, 3 veces por semana durante 22 semanas. Terrados, Valcárcel y Venta (2010) en nuevos factores de riesgo cardiovascular y la actividad física señala que el ejercicio aeróbico disminuye la concentración de TG y C-T y puede incrementar los valores del C-HDL, especialmente si se acompaña de pérdida de peso. En nuestro estudio no se efectuó control sobre la ingesta dietética, lo que origino una ganancia de peso ligeramente mayor que las ratas sedentarias para ambas dietas, no pudiendo descartar el efecto que pudo haber tenido sobre el perfil lipídico.

Entre las limitaciones de este estudio cabe citar la extensión de la piscina, que dificultaba que las ratas puedan nadar en forma homogénea, dichas ratas solo buscaban llegar al otro extremo de la piscina para quedarse ahí y descansar, mostrándose desesperadas por salir, ante el gran número de ratas empleadas para nadar se dificultó llevar un adecuado control del tiempo de nado, por lo que posiblemente unas nadaron más que otras, se recomienda emplear una piscina con mayor extensión que permita que las ratas se demoren en llegar más tiempo al otro extremo e incluso colocar especies de canales donde puedan nadar cada una sin interferir a las demás. Además, realizar un previo entrenamiento para que se adecuen al nado, trabajando periodos cortos en un inicio e ir incrementando el tiempo según vayan pasando los días de experimentación.

No se tomaron medidas iniciales. En estudios, si bien no encontraron diferencias significativas en el perfil lipídico entre sedentarios y los que practicaban alguna actividad física, si obtuvieron niveles de CT, TG, C-LDL significativamente bajos y subieron significativamente los niveles de C-HDL, en comparación a su evaluación inicial (ZapataLamana, Cigarroa, Díaz y Saavedra, 2015), por lo que se recomienda calcular el diferencial de cada parámetro lipídico. Otra diferencia marcada es que se realizaron solo siete semanas de intervención física a diferencia de otros estudios, siendo esta una debilidad para el estudio.

Se podría concluir que practicar una rutina de ejercicios aeróbicos (natación) de siete semana de duración con una frecuencia de cinco veces a la semana, 40 minutos diarios, disminuye el nivel de triglicéridos en ratas alimentadas con dieta estándar y con $2,86 \%$ de grasas trans, y que dicho programa de ejercicios aeróbico (natación), no fue suficiente para mejorar de manera estadísticamente significativa los valores de C-T, C-HDL y C-LDL.

\section{AGRADECIMIENTO}

Nuestro sincero agradecimiento al Dr. Raúl Ishiyama Cervantes, por el apoyo en la revisión del artículo. Al los doctores Reneé Pereyra Elías y Conrad Ortiz Alfaro, parte del equipo de Virtual Medical, por la asesoría en la publicación.

\section{REFERENCIAS BIBLIOGRÁFICAS}

Alfonso, J. E. F., \& Ariza, I. D. S. (2008). Elevando el colesterol HDL: ¿Cuál es la mejor estrategia? Revista da Associação Médica Brasileira, 54(4), 369-376. https://doi.org/10.1590/S010442302008000400025.

Aspíroz, M.T., \& Nuviala, R.J. (2002). Lípidos y ejercicio físico. Archivos de medicina del deporte, 19(90), 317-329. 
Ballesteros-Vásquez, M. N., ValenzuelaCalvillo, L. S., Artalejo-Ochoa, E., \& Robles-Sardin, A. E. (2012). Ácidos grasos trans: un análisis del efecto de su consumo en la salud humana, regulación del contenido en alimentos y alternativas para disminuirlos. Nutrición Hospitalaria, 27(1), 54-64.

Bermejo, L., \& Moraleda, B. R. (2013). Can the exercise mode determine lipid profile improvements in obese patients? Nutrición hospitalaria, 28(3), 607-617. https://doi.org/10.3305/nh.2013.28.3.62 84.

Camiletti-Moirón, D., Medina, G., Núñez, Á., Andrade, A., Martínez, R., Nebot, E., Kapravelou, G., Porres, J., LópezJurado, M., Aranda, P., \& Aparicio, V. (2015). Efectos de un protocolo de entrenamiento de alta intensidad sobre marcadores fisiológicos de estrés en ratas. RICYDE. Revista Internacional de Ciencias del Deporte, 11(40). R e cuperado a partir de http://www.redalyc.org/html/710/710357 85005/.

Echevarria, M. E. (2006). Relación de los niveles de apoproteínas A-I y B100 con la frecuencia de la actividad física en pacientes adultos sanos. Tesis para optar el título de Bacterióloga, Pontificia Universidad Javeriana, Bogotá.

García-Cardona, D. M., Nieto, O. A., \& Landázuri, P. (2015). Efecto del ejercicio sobre las subpoblaciones HDL, la enzima lecitina-colesterol aciltransferasa y la proteína transportadora de ésteres de colesterol en estudiantes de Medicina. Revista Colombiana de Cardiología, 22(6), 277-284. https://doi.org/10.1016/j.rccar.2015.04.0 07.

LeBlanc, A. G., \& Janssen, I. (2010). Doseresponse relationship between physical activity and dyslipidemia in youth. The Canadian Journal of Cardiology, 26(6), e201-e205.

Monrroy, R. (2009). Ácidos grasos trans: riesgos a la salud y legislación mexicana. Ide@s Concyteg, 4(49), 767778.
Poveda, E., Ayala, P., Rodríguez, M., Ordóñez, E., Baracaldo, C., Delgado, W., \& Guerra, M. (2005). Efecto del suplemento de aceites vegetales sobre el perfil lipídico en ratas wistar. Biomédica, 25(1), 101-9.

https://doi.org/10.7705/biomedica.v25i1 .1331.

Salgado Ortega, M. L., Rivera, A.E., Habacuc, M., Sanchez-González, J., Macera, A., Aboytes, M.J., \& Tovar, L. (2003). Influencia de la grasa corporal y el sexo sobre la respuesta de los lípidos séricos al ejercicio físico en personas con diferente capacidad aeróbica. Revista mexicana de patología clínica, 50(2), 5870 .

Souki, A., Vargas, M. E., Gabarrón, J. M., Escalona, D., Aguirre, M., Matta, M., Almarza, J., Barroso, E., Medina, M., \& Cano, Cl. (2007). Adición de mayonesa a la dieta de rata Sprague-Dawley incrementa la glicemia y los triacilglicéridos con disminución del HDL-colesterol. Archivos Venezolanos de Farmacología y Terapéutica, 26(1), 66-69.

Terrados, N., Valcárcel, G., \& Venta, R., (2010). Los nuevos factores de riesgo cardiovascular y la actividad física. Apunts Medicina de Esport, 45(167), 201-208.

Wong-On, M., \& Murillo-Cuzza, G. (2004). Fundamentos fisiopatológicos de la obesidad y su relación con el ejercicio. Acta Médica Costarricense, 46, 15-24.

Zapata-Lamana, R., Cigarroa, I., Díaz, E., \& Saavedra, C. (2015). Reducción del riesgo cardiovascular en mujeres adultas mediante ejercicio físico de sobrecarga. Revista médica de Chile, 143(3), 289-296.

https://doi.org/10.4067/S003498872015000300002

\section{Correo electrónico:}

gba_23@hotmail.com

Revisión de pares:

Recibido: 20-04-2017

Aceptado: 28-06-2017 